Vector Grammar, Places, and the Functional Role of the Spatial Prepositions in English

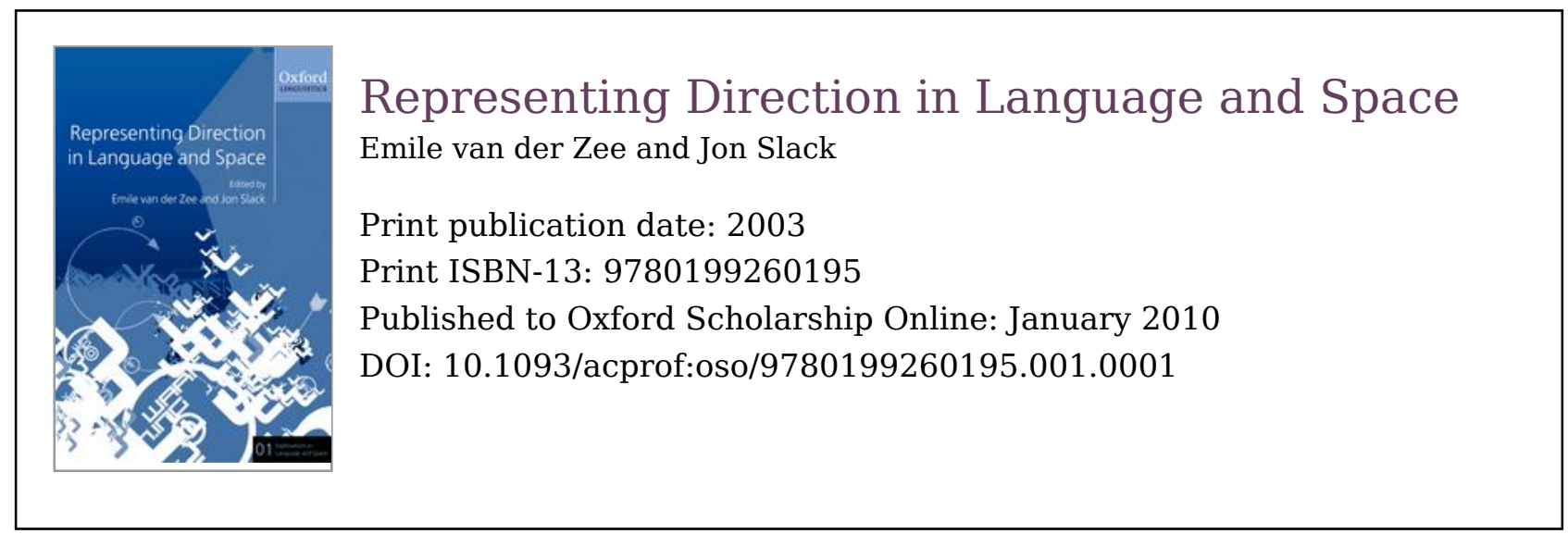

\title{
Vector Grammar, Places, and the Functional Role of the Spatial Prepositions in English
}

\author{
JOHN O’KEEFE
}

DOI:10.1093/acprof:oso/9780199260195.003.0004

\begin{abstract}
Keywords
This chapter shows how different prepositions specify different aspects of the location vectors and of the spatial extents of the fields in different directions. Animal studies provide evidence that vector-based representations are employed in spatial cognition. This work addresses the issue of how different spatial relations are encoded within a vector-based representation called a cognitive map, which is an absolute or allocentric spatial representation of the environment. This chapter introduces the Boundary Vector Cell model, which assumes that place cells - representing certain locations in space-take their input from Boundary Vector Cells (BVCs). The properties of the BVCs and place cells cooperate to form graded regions; regions that are not isotropic, but represent a continuous acceptability gradient for many different spatial prepositions.
\end{abstract}

Keywords: spatial prepositions, location vectors, direction, grammar, cognitive maps, Boundary Vector Cell, place cells, space, spatial cognition
Abstract
In a previous work, I presented a theory of the role of the locative prepositions in English which suggested that their primary function was to identify a set of vectors that located regions in a Narrative Map, a device for storing narratives and other linguistic representations. In this chapter, I present an updated version of this idea. Here I draw upon recent computational ideas generated by our efforts to model the firing fields of the spatially coded place cells in the rat hippocampus. The chapter attempts to show how different prepositions specify 
Vector Grammar, Places, and the Functional Role of the Spatial Prepositions in English

different aspects of the location vectors and of the spatial extents of the fields in different directions.

Introduction

I wish to update my proposal (O'Keefe, 1996) that the neural basis for language might be a structure termed a Narrative Map in which syntactic relationships are specified by places, the names of the objects that they contain, and the vectors connecting them. This is a proposal that Lynn Nadel and I first mooted in our book The Hippocampus as a Cognitive Map (1978) and which is motivated by neural as well as linguistic considerations. We have proposed that the hippocampus, a cortical area in the mammalian forebrain, is involved in the construction of an allocentric spatial representation of the environment, what (p.70) Tolman (1948) called a 'Cognitive Map'. In animals, this map is purely or primarily spatial: the hippocampal formation provides the animal with a representation centered on the environment which enables it to locate itself and objects of interest within that environment. In humans however, the hippocampus is necessary for the storage and recall of linguistic and episodic memories as well as for spatial memories and so it was necessary to extend the basic theory to account for the human data. We assume that the right human hippocampal formation continues to have a primarily spatial function, operating in much the same way as the rat hippocampus. The left hippocampus, on the other hand, has been modified in two ways to transform it into a linguistic/ episodic memory system. Firstly, this spatial structure has acquired an extra dimension enabling it to incorporate a temporal sense into the basic map to account for the ability of humans to process and store spatio-temporal information. We argue that this framework provides the basis for episodic memory, which is the ability to recall personally experienced events set within their original spatiotemporal context. Secondly, we argue that the primary input to the left hippocampal formation consists of information about linguistic entities rather than about physical objects referred to the external physical world. For example, damage to the left mesial temporal lobe usually results in impairment in the memory for linguistic material and in particular words and narratives (see e.g. Frisk and Milner, 1990). I will briefly outline our current understanding of the neural elements which contribute to the Cognitive Map and then I will sketch our recent computational model of the formation of the place representations that form the core of the Cognitive Map and show how some aspects of this model relate to the prepositions in English.

\section{Cognitive Map Theory}

The Cognitive Map consists of a set of place representations and their spatial relationship to each other, objects are located by their relationship to places in the map and only indirectly by their spatial relationships to each other. Spatial relationships are specified in terms of three variables: places, directions, and distances. Places are patches of an environment that can vary in size and shape depending on the size of the environment and the distribution of features in that 
environment. They are located in two ways: first, in terms of the spatial relations amongst the invariant features of the environment and second and independently, by their direction and distance from other places. The place code is carried by the pattern of firing of the place cells in the cortical region called the hippocampus. Directions are specified as a set of parallel, infinitely (p.71) long vectors. As with places, these can be identified in one of several ways: either as the local gradient of a universal signal such as gravity, geomagnetism, or olfactory currents, or as the vector originating at a place or object and passing through another place or object (or passing through two places), or as having a specified angle to a previously identified direction (e.g. through updating the current direction on the basis of angular head movements). For every direction there is an opposite direction which can be marked by the negative of that vector. The direction code is carried by the pattern of firing of the head direction cells, which are located in several brain regions but most notably in the dorsal presubiculum (see e.g. Taube et al., 1990), a cortical region that neighbors on the hippocampus and is anatomically connected with it.

Distances between objects or places are given by a metric, which can be derived in one of two ways. Ultimately distances are based on a signal that converts information about the organism's movements in an environment into the speed with which those movements translate the animal through the environment. The integral of this speed signal is combined with introceptive (e.g. vestibular) and/or environmental (e.g. directional) information to calculate a distance between places. The changes in the relative locations of objects as a result of movements in the environment can also be used to compute distances.

A path is defined as an ordered sequence of places and the direction vectors between them. Paths can be identified by their end places or, in humans, by a distinct name. Conversely, places along the path can be identified and associated with the path. A path may be marked by a continuous feature such as an odor trail or a road, but need not be.

Within this spatial framework, translations of position in an environment are specified as translation vectors whose tail begins at the origin of movement and whose head ends at the destination. Vector addition and subtraction allow journeys with one or more subgoals to be represented and integrated.

Furthermore, on a journey with more than one destination the optimal or minimal path can be calculated. In recent computational work, our group has suggested that the activity patterns of the cells which encode the place representation might be constructed on the basis of inputs that identify a location on the basis of its direction and distance from large environmental features such as a wall (Hartley et al., 2000). Here I will pursue a variant of this idea and see whether the spatial prepositions can be identified with different aspects of this computational model. 
Vector Grammar, Places, and the Functional Role of the Spatial Prepositions in English

I have previously argued that an understanding of sentences about physical space might provide an important insight into the deep semantic structure of language in general. There is a long tradition in linguistics, revived within Case Grammar theory, which postulates that the deep semantic structure of (p.72) language is intrinsically spatial and that other, non-spatial, prepositions are in some way parasitical on these prototypical formulae, perhaps by means of metaphorical extension of their core spatial meanings. This is the contention of a group of linguists called locationists or localists (Anderson, 1971; Bennett, 1975) (see Cook, 1989, for a review). In an uninflected language such as English many of the spatial relations described in spatial sentences are conveyed by the prepositions. A description of the representations set up by the spatial prepositions might provide the basis for a more general linguistics. Nadel and I opined that the origin of language might have been the need to transmit information about the spatial layout of an area from one person to another (O'Keefe and Nadel 1978: 401 n.), perhaps as adjuncts to simple maps used to convey the location of food items or dangers to other family members. Originally the linguistic content of these prototypical Semantic Maps might be rather simple and impoverished. Different sounds might stand for different objects in the map and might serve the additional function of acting as an encrypting device. Over time the pictorial aspects of the structure of the map might be systematically replaced by prepositional and other spatial semantic elements. This increase in syntactic vocabulary would eventually obviate the need for the externalized map entirely but the neural substrate would retain the underlying map-like structure of the original.

In O'Keefe, 1996, I set out the basic framework of Vector Grammar and showed how it accounted for many of the spatial meanings of the spatial prepositions. My thesis was that the primary role of the prepositions was to provide the spatial relationships among a set of places and objects, and to specify movements and transformations in these relationships over time. These spatial relationships and their modifications were viewed as represented by vectors. A similar idea was proposed by Zwarts (1997) around the same time.

In Vector Grammar Theory, the location of an entity within this notation is by a vector, which consists of a direction and a distance from a known location. Some of the work of the locative prepositions involves the identification of aspects of these variables. In some cases (e.g. with vertical prepositions such as below), the direction is given by an environmental signal such as the force of gravity. In most cases, however, it needs to be calculated from the spatial relationships between two or more objects or places. In these latter cases the prepositions specify the origin and termination (or the tail and the head) of the vector or a point along the vector. In contrast, distances are less well specified; in most cases the metric is an interval one. This reflects the fact that many prepositions describe relationships that are transitive, linearly scalable, and insensitive to absolute location. If $\mathrm{A}$ is above $\mathrm{B}$ and $\mathrm{B}$ is above $\mathrm{C}$, then it follows that $\mathrm{A}$ is above 
Vector Grammar, Places, and the Functional Role of the Spatial Prepositions in English

C. Doubling the distance between A and B, and between (p.73) B and C does not change any of these relationships. Nor does shifting all three entities a constant distance in the same direction. One of the roles of the preposition for is to supply the necessary metric information. When this is available, the relationships described by the spatial prepositions can attain the level of a ratio scale (for further discussion of these ideas, see O'Keefe, 1996). The 3-D space coded by the locative prepositions is a mixed polar-rectilinear one.

In this chapter I shall explore a modified version of the same vector-based approach. Many of the ideas it contains are derived from extensions of the recent work of our group in which we have proposed computational models for the place fields and their cortical inputs. In this work we have suggested that places can be modeled as the sum of two or more gaussians. The center of each gaussian is located by its distance in a particular direction from an environmental feature, i.e. a vector. Several of these gaussians are combined in the hippocampal formation to form a place representation. It is my contention that some of the prepositions code for aspects of single directional vector gaussian inputs to the hippocampus whereas others seem to require more complex representations anchored in several directions, perhaps even ones similar to the place cells themselves. I assume (following the locationists, see above) that the prepositions in English have a spatial (or in one or two instances temporal) sense as their basic meaning and that the other meanings are derived by metaphorical extension. Although the theory is intended to extend eventually to all prepositions, spatial, and non-spatial, here I will concentrate on the locative prepositions and, in particular, those which deal with the space around the reference object or place. On the basis of the place cell model, I will categorize the spatial prepositions into those that locate the vector components and those that locate aspects of the gaussian components or the boundaries of the field. When speaking of the strictly spatial meaning of the prepositions, I will refer to the area under the intersection of the gaussians as the place field. In the context of the broader non-spatial meanings of the prepositions, I will refer to this area as the semantic field.

\section{A Computational Model for Place Representations}

Single neurons in a cortical structure in the rat brain called the hippocampus become active when the animal visits a patch of a familiar environment.

Different place cells have different preferred patches or place fields in the same environment. Experiments in which the place fields of the same neurons were recorded in different-shaped rectilinear boxes suggest that each field is the composite of two or more subcomponents, each of which is fixed to a large feature (p.74) of the environment such as a wall of the room or the holding box (O'Keefe and Burgess, 1996). On the basis of these experiments, we have suggested that each subcomponent of a place field might reflect a separate input coming from cortical structures afferent to the hippocampus. In subsequent work, we have generalized this model so that it applies to environments and 
Vector Grammar, Places, and the Functional Role of the Spatial Prepositions in English

features of different shapes and can be used to predict the response of each cell in a wide variety of environments (Hartley et al., 2000). These putative inputs are termedm Boundary Vector Cells (BVCs). BVCs fire as a function of the distance of the animal in a specific direction from a large environmental feature such as the wall of the holding box. The model states that each place cell has inputs from two or more BVCs. It is with different aspects of a generalized version of these putative BVC inputs that I wish to identify different spatial prepositions. The basic idea is that each of the spatial prepositions sets up a different spatial field and these are subsequently combined in the hippocampus to form complex structures that underpin the interpretation of episodes, narratives, and narrative memories. The equation for the field of a putative BVC input is

$\varphi_{\mathrm{i}}$ is the angle of the direction vector in polar coordinates relative to a reference direction. This reference direction can be determined in several different ways but in general is fixed to an environmental frame rather than to an egocentric frame;

$d_{i}$ is the distance of the center of the field from the origin

(1) $\quad g_{i}(r, \theta) \propto \exp \left[-\left(r-d_{i}\right)^{2} / 2 \sigma_{\operatorname{mad}}^{2}\left(d_{i}\right)\right] / \sqrt{2 \pi \sigma_{\operatorname{rad}}^{2}\left(d_{i}\right)}$ $\times \exp \left[-\left(\theta-\varphi_{i}\right)^{2} / 2 \sigma_{\text {ang }}^{2}\right] / \sqrt{2 \pi \sigma_{\text {ang }}^{2}}$

The receptive field is a function of four parameters: along the direction vector $\varphi_{i}$;

$\sigma_{\text {rad }}$ is the width of the field in the radial dimension; while

$\sigma_{a n g}$ is the width in the angular dimension.

In the cognitive mapping system of the rat, the location of the BVC field is given by the length of the direction vector (i.e. a distance in a particular direction) from the animal itself (see Fig. 4.1).

The size and shape of the BVC field are given by the distance from that landmark in the radial direction and by a fixed angular width in the other. This results in the fields close to the rat being smaller and more strongly peaked than those farther away. In the human linguistic system, an analysis of the prepositions suggests that the fields are sometimes located relative to the (p. 75) speaker or listener but more generally relative to other objects in the environment (the reference landmarks). Furthermore it would appear that the size and shape of the semantic field can be specified independently of its distance from the reference landmark. As we shall see in the subsequent sections, some prepositions (e.g. beyond and below) identify only one of the radial borders of the field while others (e.g. behind and under) additionally specify the two angular boundaries, and still others (such as between, among, 
Vector Grammar, Places, and the Functional Role of the Spatial Prepositions in English

and in) delimit all four boundaries. These latter can the thought of as formed by the intersection of several simple Boundary Vector fields and as much closer to the fully-fledged place fields found in the hippocampus of the rat. In the cognitive mapping system of the rat, the direction vector is usually given by environmental information or internal signals derived primarily from the vestibular system to orient the sense of direction. In humans, the direction vector is given by the universal gravitational signal in the $\mathrm{Z}$ direction and is calculated in the horizontal XY plane by reference to two or more objects in the environment. Often one of these objects is the speaker or listener (i.e. a deictic origin). In the rat mapping system, the source of distance information is not known for certain but probably involves the integration over time of path integration signals derived from the animal's movement or the use of exteroceptive (e.g. visual) cues from familiar reference objects. In the human vector grammar system, distances are sometimes given by modifiers of the prepositions. These can be given as relative distances such as farther than or absolute distances such as $3 \mathrm{ft}$.

(3) John was farther behind the house than Jim.

(4) John was $3 \mathrm{ft}$. behind the car.

In general these modifiers act upon the length of the vector specified by the preposition, either in comparison to another vector or by specifying an absolute (p.76) length. It does not seem possible to modify the other parameters specified by the prepositions such as the width or length of the place field.

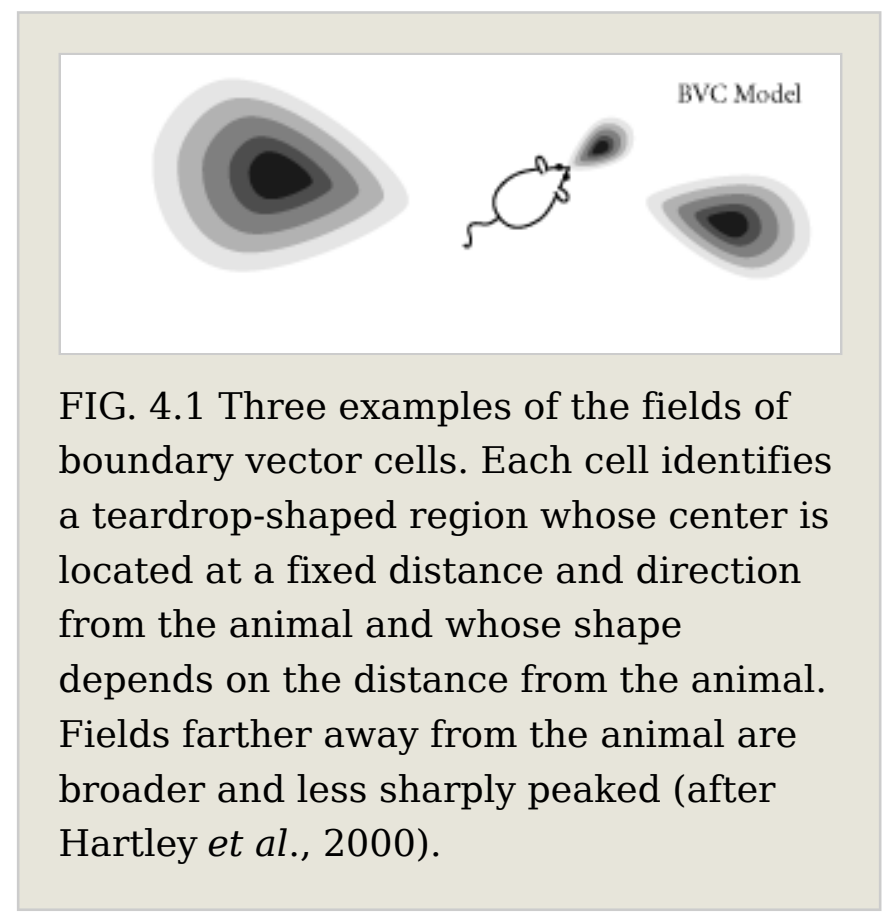

\section{The Semantics of Selected Prepositions}

As examples of this approach, I will discuss the prepositions beyond, below, behind, under, by, beside, and, finally between, in, and among.

\subsection{Beyond and Below}

In O'Keefe (1996) I suggested that the meaning of beyond could be captured by the set of vectors whose length bore a specific relationship to a reference vector drawn to the distal surface of the reference object. Specifically it consisted of all vectors whose inner product when projected onto the direction reference has a larger magnitude than a reference vector drawn from the origin to the far end of 
Vector Grammar, Places, and the Functional Role of the Spatial Prepositions in English

the reference location or object. No restrictions were placed on the radial or angular width of the terminations of the set of vectors specified. Usually these would be expected to be larger than the width and length of the reference object. While the proximal boundary of the vector field was well specified, the distal boundary was left open. Modifiers such as just or far place restrictions on the length of the semantic field vector in relation to the distance to the landmark.

(5) The barn is just beyond the lake.

(6) The barn is far beyond the lake.

The role of the comparative modifier farther is to increase the distance $\delta$ and thus the location of the semantic field. It also has the subsidiary effect of placing an upper limit on the distal boundary of the secondary semantic field (in the example below, that of the house).

(7) The barn is farther beyond the lake than the house. $\delta_{b}>\delta_{h}$

Below has a meaning analogous to beyond in the vertical direction. In O'Keefe (1996) I gave its meaning as the field of vectors whose distance along the vertical reference vector was more negative than that of the reference object.

One of the problems with this use of a vector field to designate the place fields located by prepositions is that it describes a homogeneous field within which the locandum has an equal probability of being found at every location. While this may be an accurate depiction of the semantic field of beyond for (p.77) some speakers of English, it does not accord with my own intuitions, which suggest some internal structure to the fields of beyond and below such as that represented in Figs. 4.2 and 4.3. This intuition is even stronger in the case of other related but more complex prepositions such as behind and under (see below). 
Vector Grammar, Places, and the Functional Role of the Spatial Prepositions in English

\begin{tabular}{|c|c|}
\hline $\begin{array}{l}\text { (p.78) It is obvious that the } \\
\text { structure of the semantic fields } \\
\text { shown in Figs. } 4.2 \text { and } 4.3 \text { bears } \\
\text { a strong resemblance to those } \\
\text { of the BVC cells in Fig. } 4.1 \text {. One } \\
\text { interpretation of the semantic } \\
\text { field idea is that it describes the } \\
\text { probability of finding the } \\
\text { located object within the field, } \\
\text { in this case highly likely close to } \\
\text { the center and less likely as one }\end{array}$ & $\begin{array}{l}\text { located. The center of the field is located } \\
\text { by a point along the direction vector } \mathrm{V} \text { at } \\
\text { a distance } \delta \text { from the origin (in this } \\
\text { example the Observer). Distance } \delta \text { is } \\
\text { greater than } \delta_{\mathrm{f}} \text { the distance from the } \\
\text { origin to the farthest edge of the } \\
\text { reference object (shown as a grey block). } \\
\text { The length and width of the place field } \\
\text { are left unconstrained and the width may } \\
\text { be larger than the width of the reference } \\
\text { object. }\end{array}$ \\
\hline
\end{tabular}

moves towards the periphery.

Empirical support for the idea that there is structure to the space identified by prepositions such as below comes from the work of Logan and Sadler (1996). They showed students two letters on a video screen, a centrally placed $\mathrm{O}$ and an $\mathrm{X}$ at one of 49 locations, and asked them to rate how acceptable on a scale of 1 (least acceptable) to 9 (most acceptable) the location of $\mathrm{X}$ was as an exemplar of the sentence 'the $\mathrm{X}$ is [relation] the $\mathrm{O}^{\prime}$. Among the relationships tested were above and below. The average acceptance profiles for each location of $\mathrm{X}$ for the relations below (A) and above (B) are plotted as contour maps in Fig. 4.4.

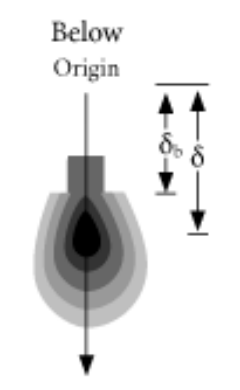

FIG. 4.3 The place identified by the preposition below has its center on the vertical direction vector drawn through the reference object at a distance $\delta>\delta_{b}$, the lower edge of the reference object. Like the field of beyond, there is no restriction on the width of the field in the orthogonal horizontal plane.

While Logan and Sadler divide the fields into three regions, good, bad, and acceptable, in line with their spatial template model, it is clear that the field might be better described as a continuous region peaked in the center and falling off gradually in a monotonic fashion in all directions. These fields look remarkably similar to the teardrop-shaped fields shown in Fig. 4.3. Further studies of this nature will be necessary to identify the exact mathematical (p. 79) functions associated with each preposition but the general correspondence to the proposed model is encouraging. 
Vector Grammar, Places, and the Functional Role of the Spatial Prepositions in English

3.2. Behind and Under

In O’Keefe (1996), I defined behind as the set of vectors with a larger magnitude then a reference vector but with an angle less than or equal to the vector drawn to the outer edge of the reference object. Within the current framework, behind is defined as shown in Fig. 4.5.

The field is the same as that for beyond except that the width is restricted by the angle $\varphi$. This is the angle made by the vector drawn from the origin to (i.e. along) the outer edge of the reference object. The field structure is such that there is a greater likelihood of finding the locandum towards the center of the field and less likelihood as one moves towards the edges.

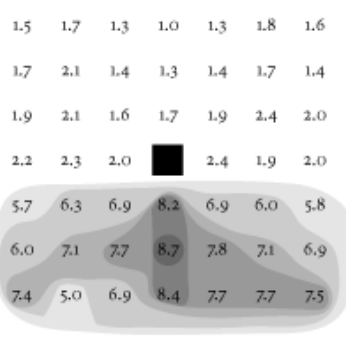

(a)

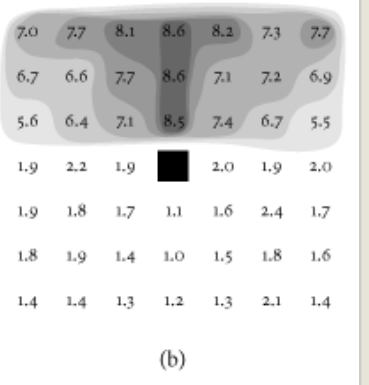

(b)
FIG. 4.4 (a): Acceptability ratings for locations in terms of how well they satisfy the relationship below the $O$ (marked by the grey square here). Higher numbers denote greater acceptability. Contour maps have been superimposed on the lower half of the figure to provide an idea of the semantic field of the preposition below. (b): Acceptability ratings for the preposition above. Contour levels used in both plots are 8.5, 7.5, 7.0. 6.0, and 5.0. Modified from Logan and Sadler (1996) with permission. See original paper for additional details.

The preposition under was

previously viewed as having two meanings; one similar to that of below and a second slightly more restricted one. This second meaning differed from that of below in that it further restricted the region in the horizontal plane to the projection of the reference object onto that plane. In the model presented in this chapter, the region of below (and under ${ }_{1}$ ) is (p.80) identified as shown in Fig. 4.3, and that of under 2 in Fig. 4.6. The center of the field of under 2 is given by the distance $\delta_{d}$ from the origin of the vertical direction vector and $\delta_{b}$, the distance from the edge of the field. The boundaries of the field and the extent of the region under in the XY plane are determined by vectors parallel to the vertical direction vector and intersecting the sides of the reference object. 
Vector Grammar, Places, and the Functional Role of the Spatial Prepositions in English

The importance of distances in the horizontal XY plane is illustrated by the operation of the modifier farther in this dimension (cf. Fig. 4.7b).

(8) The white box is farther under the shelf than the white circle.

Notice that the interpretation of this sentence depends on whether the shelf is located against a wall or not. When the reference object is such that it can (p.81) be approached from either side then the location of the peak of under would appear to be determined by the center of the shelf. If, however, approach is only possible from one end, as in Fig. 4.7c where the shelf is fixed to a wall, then the peak of the field is close to the wall. This is a good demonstration that the field need not be symmetrical nor need the field peak be located in the geometrical center of the field.

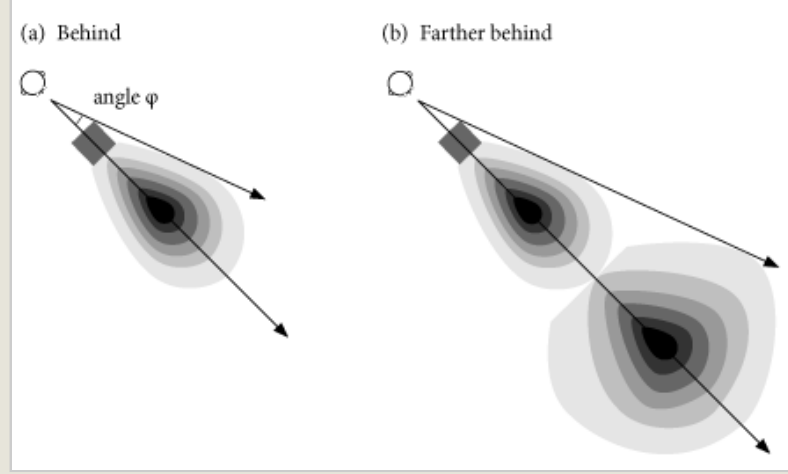

FIG. 4.5 (a): The place identified by the preposition behind is the same as that of beyond except for greater restrictions on the axial width of the field. The width of the field is determined by the vector with angle $\varphi$ to the direction vector. (b): The semantic field of farther behind.

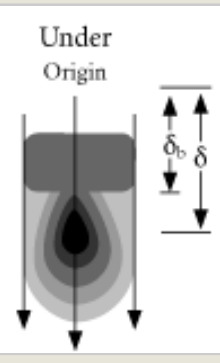

FIG. 4.6 The place identified by the preposition under is the same as below except that the width of the field in the horizontal plane is restricted by vectors drawn parallel to the vertical reference vector through the outermost edges of the reference object.

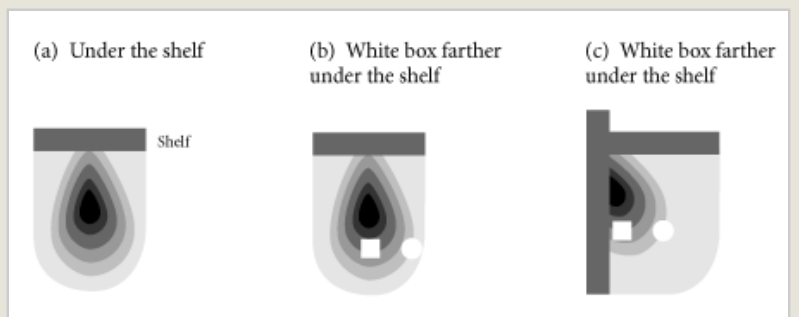

FIG. 4.7 (a) shows the field of under the horizontal shelf. In both (b) and (c), the 
Vector Grammar, Places, and the Functional Role of the Spatial Prepositions in English

The action of comparators such as farther in Fig. 4.7 is different from the effect of these modifiers on the preposition below (and under 1 when it is used with reference objects which have very large or unlimited extents in the horizontal plane) where they appear to act on the length of the vector $\delta$ in the vertical direction.

(9) Farther below the surface of the lake (under the water).

It is of interest that the subjects in the experiments of Logan and Sadler (American college students) did not distinguish between below and under, suggesting that they were restricting their usage to the first meaning of under. This may have been due to the use of relatively small letters as the objects to be located in this experiment. It would be interesting to see if a distinction between below and under emerges when horizontally extended reference shapes are used.

\subsection{By and Beside}

$B y$ is shown in Fig. 4.8. The region designated by by or part of this region can also be identified by the preposition beside. Whether the whole of the by field, or only part of it, is considered to be beside the reference object depends on (p.82) the availability of a reference vector in the orthogonal XY plane to polarize the region into before and behind subcomponents. In the absence of a horizontal reference vector, the entire region is beside the reference object. When it is present, the region designated by by can be further subdivided into four continuous regions, one in front of or before it, one behind it (see above), and two beside it. The beside field is restricted to that part of the by region which is neither before nor behind the reference object. This means that beside involves a third-order computation, depending on the prior identification of the above and below regions followed by the front and back regions. This may explain why the speed with which these judgements can be made about the location of an object are fastest for above and below, followed by front and back, and slowest for left and right (beside) (see Tversky, 1996). This analysis would seem to suggest that an object cannot be both behind and beside a reference object at the same time.

(10) *The car beside and behind the house

For a small number of objects (most notably humans, animals, furniture), the usual orientation of the reference vector leads to their being assigned a front, back, and sides, especially when

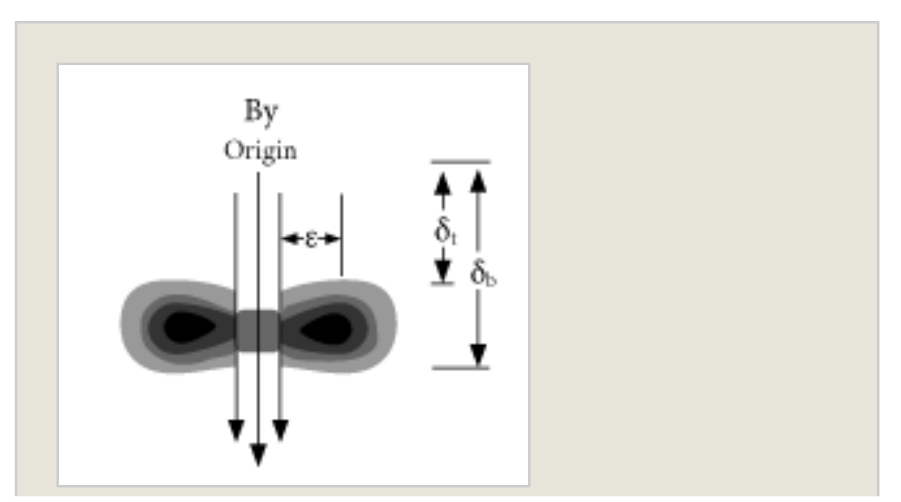


Vector Grammar, Places, and the Functional Role of the Spatial Prepositions in English

\section{they are in their canonical orientations. This has led to the idea that these locations are determined by the major axes} of the object (Landau and Jackendoff, 1993). This might tempt us to think that these prepositions refer to space that is fixed to the objects themselves. However, an alternative interpretation is that, in appropriate contexts, the axes of the object determine the orientation of the direction vectors. For example, for a human this would mean that the canonical direction vector for behind would have an orientation pointing from the person's chest to his or her back. However, these canonical interpretations are easily overridden in non-standard situations, for example, when the reference object is in an unusual orientation.

(11) the empty bottle in front of the upturned chair

(12) Stand beside that man lying on the floor.

It should be further noted that the ability to identify canonical orientations depends on the availability of vertical, and in some cases horizontal, reference vectors in the first place.

\subsection{Between, In, and Among}

These are the prepositions that come closest to defining fully-fledged place fields. They specify not only the location of the field center but also its boundaries on all sides. They differ in the way in which the field boundaries are specified. (p.83) Between specifies two reference objects which determine the boundaries, among three or more objects which locate the perimeter of the field, while in identifies an object or a location the boundary of which coincides with that of the field. Figure 4.9 shows the field of between. The center of the field is located on the line joining the centers of the two reference objects and the boundaries are formed by (1) the surfaces of these objects facing each other and (2) by the lines connecting the outer surfaces of the reference objects. The field structure suggests that objects located closer to the line joining the midlines of the two reference objects will be perceived as better instantiations of between than those closer to the periphery. 
Vector Grammar, Places, and the Functional Role of the Spatial Prepositions in English

The field of in is bounded by the inner surface of the reference object with a peak somewhere near the centroid. Note that the field of in need not necessarily be coextensive with the reference object that defines its boundary, in particular where the reference object is not a continuous one. For example, the field of a bowl that is open at the top may continue to follow the extrapolated curvature of the bowl and not the object itself and objects located in this region would still be in the bowl.

\subsection{Synecdoche and the Semantics of the English Prepositions}

The strong geometric interpretation of the prepositions taken in this chapter would seem to invite the criticism that it gives an overly precise interpretation of their meanings. It should be remembered however that the place fields represent probability distributions within which objects may be found and do not specify precise locations as such. Furthermore, my view is that some of the exceptions and ambiguities that might be used to challenge this view can be (p. 84) answered by a closer look at the way in which synecdoche and ellipsis influence the interpretation of prepositional phrases.

(13) flowers in the vase

(14) chair under the table

Both phrases clearly represent the use of the whole for the part. In the first sentence it is the stems of the flowers that are in the vase, while in the second sentence it is usually the seat of the chair that is under the horizontal surface of the table (and not e.g. the back). I suspect that some of the functional interpretations of the meanings of the prepositions may yield to a similar analysis.

\section{Summary}

1. Cognitive Map Theory suggests that the function of prepositions is to locate place fields within a Semantic or Narrative Map, the linguistic analog of a spatial map.

2. Semantic Maps are devices for storing the relationships between linguistic entities such as nouns in the form of narratives, including the personal narratives or stories that conscious subjects construct about their experiences. 
Vector Grammar, Places, and the Functional Role of the Spatial Prepositions in English

3. In previous work I have suggested that entities within the map were located by sets of vectors. Here I offer a more natural representation in terms of place fields analogous to those found in the hippocampus of the rat. These fields are identified by their center and spread in two or three orthogonal dimensions.

Simple semantic fields resemble those we have modeled as Boundary Vector Cells. They are located at the end of a vector and have relatively fixed boundaries that depend on the distance from the reference object. More complex semantic fields are closer to true place fields and may be located by the intersection of two or more simple fields, each determined by a vector in a different direction. Unlike the previous vector field theory, which gave a uniform probability of locating an object in an area, the current place field theory provides internal structure to the area located by the preposition and suggests that the target to be located is more likely to be found in certain parts of the field than in others.

4. Field Centers are located at the termination(s) of one or more principal vectors. The orientation of the principal vertical direction vector is usually provided by the universal gravity signal. On the other hand, horizontally oriented vectors need to be computed on the basis of a vector orthogonal to the horizontal vector or to the relative positions of landmarks, usually by vector subtraction of the vectors from the observer to two of these landmarks.

(p.85) 5. Some prepositions (e.g. beyond, below, and above) identify regions that are relatively unrestricted in the plane orthogonal to the primary location vector. For this class of prepositions, comparative modifiers such as farther or near operate to increase or decrease the length of the principal vector. Other prepositions such as behind, by, and beside place further restrictions on the boundary of the field. Prepositions such as between, in, and among identify the boundaries of the field completely.

\section{Access brought to you by:}

